\title{
Thermal Properties of an Inflationary Universe
}

\author{
Arjun Berera \\ Department of Physics, Pennsylvania State University, University Park, PA 16802, U.S.A.
}

\begin{abstract}
An energetic justification of a thermal component during inflation is given. The thermal component can act as a heat reservoir which induces thermal fluctuations on the inflaton field system. We showed previously that such thermal fluctuations could dominate quantum fluctuations in producing the initial seeds of density perturbations. A Langevin-like rate equation is derived from quantum field theory which describes the production of fluctuations in the inflaton field when acted upon by a simple modeled heat reservoir. In a certain limit this equation is shown to reduce to the standard Langevin equation, which we used to construct "Warm Inflation" scenarios in previous work. A particle physics interpretation of our system-reservoir model is offered.

PACS numbers: 98.80.Cq, 05.40.+j
\end{abstract}

In press Phys. Rev. D 1996

hep-th/9601134 


\section{INTRODUCTION}

According to inflationary cosmology, the large scale structure of the present day universe is essentially a kinematic outcome of exponential amplification of perturbing seeds in an initially smooth universe [1-3]. The differential microwave radiometer (DMR) on the Cosmic Background Observer (COBE) has made the first direct probe of the initial density perturbations through detection of the temperature anisotropies in the cosmic background radiation $(\mathrm{CBR})$. These results are consistent with the scaling spectrum given by the inflation model. They also reinforce previously known measurements, although done by less direct methods, that the amplitude of initial perturbations is

$$
\Delta(\mathbf{k}) \equiv \frac{\delta \rho(\mathbf{k})}{\rho}=10^{-3}-10^{-5}
$$

and approximately constant for all wavenumber $\mathbf{k}[$ [4,5]. Here $\rho$ is the energy density in the present universe and $\delta \rho(\mathbf{k})$ is its rms-deviation at wavenumber $\mathbf{k}$.

We can understand the underlying kinematic origin of large-scale structure formation through a picture. Let us imagine observing the universe before inflation. It is a small patch that is growing at light speed. The process of inflation can be thought of as a rapid stretching of this patch in all directions. Comoving and physical coordinates are useful for further description. Comoving coordinates stretch with the patch so do not change for points that are stationary with respect to the patch. Physical coordinates express distances in terms of a physical measure, such as the local speed of light. A physical coordinate system is defined locally to a given point on the patch. It is useful sometimes to understand global distances in terms of physical units. For definitiveness, at the onset of inflation, let the comoving coordinates coincide with the physical coordinates. If we imagine the universe to be spherical with radius $R_{0}$ at the onset of inflation, then during inflation, in comoving units, the radius remains the same whereas in physical units it grows as $e^{H t} R_{0}$. Thus during inflation points on the preinflationary patch that are stationary in the comoving frame will rapidly move apart in terms of physical coordinates.

Identify a point a on the preinflationary patch. Suppose that before inflation instruments are placed at $\mathbf{a}$, which maintain communication with all points within reach by light since point a was created in the Big Bang. As inflation begins, communication is first lost with points furthest away from a. Immediately after inflation, only points that were initially very close to a will remain in communicative contact. To be definitive, just before inflation let the most distant points communicating with a be some distance $L_{0} \sim 1 / H$. Here $H$ is the Hubble constant with $1 / H=5 \times 10^{-11} \mathrm{GeV}^{-1}=10^{-23} \mathrm{~cm}=3 \times 10^{-35} \mathrm{sec}$. The time interval $1 / H$ is typically referred to as an e-fold.

To understand the behavior of physical measures during inflation, let the origin of cosmic time, $\mathrm{t}=0$, correspond to the beginning of inflation. The physical distance of a comoving interval $\Delta x$ at time t will then be $e^{H t} \Delta x_{p h y s}$. On the other hand, for a light signal emitted at $t=0$, computing along its geodesic $d s^{2}=0$, it will travel a physical distance $\left(e^{n} / H\right)\left(1-e^{-n}\right)$ after $\mathrm{n}$ e-folds of inflation.

The implication of these two relations to physical correlations can be understood from the following example. For a signal emitted at $t=0$, points fixed with respect to the comoving coordinates (comoving points) that are less than a comoving distance $(1 / H)\left(1-e^{-1}\right)$ will 
receive this signal before the first e-fold of inflation. However comoving points greater than a comoving distance $1 / H$ from a can never receive the light signal within the inflation stage. Only a sufficient time after inflation could such points communicate with a. In terms of physical distances, one concludes that comoving points that are greater than a physical distance $1 / H$ at $t=0$ will lose communicative contact with a all during inflation. Since nothing is special about point a to any other point on the patch, on the large scale physical distances at $t=0$ that are greater than $1 / H$ will act incoherently all during inflation. Thus correlations larger than $1 / H$ physical units are thereafter "frozen". This phenomenon is sometimes referred to as freeze-out or horizon crossing [3].

If a emits a second light signal at the end of the first e-fold, by the end of the second e-fold this signal again will have traveled a physical distance $(e / H)\left(1-e^{-1}\right)$. However comoving coordinates will have stretched by a factor $e^{2}$ in terms of physical units. Thus only those points which are a comoving distance $e^{-1} / H$ or less can receive the second signal at sometime within the inflation stage. Expressed in physical units, the conclusion stated above from the first e-fold repeats itself for the second e-fold: comoving points that are greater than a physical distance $1 / H$ at $t=1 / H$ hereafter will lose communicative contact with a all during the rest of inflation. Finally a signal emitted from a at the end of the n-th e-fold can be received by comoving points within a comoving distance $e^{-n} / H$ from a. Thus comoving points greater than a physical distance $1 / H$ at $t=n / H$ thereafter lose communicative contact with a during the rest of inflation.

Any local energy perturbations during inflation can affect a region of characteristic physical length $1 / \mathrm{H}$ or less. The earlier a given perturbation occurs, the more elongated it will become due to inflation. As such the largest scales of energy density fluctuations in the post-inflationary universe arose from the earliest perturbations during inflation.

In the standard scenario [6], inflationary expansion is assumed to occur within a supercooled universe, in which the initial energy density perturbations were produced by quantum fluctuations. However energetics does not require a supercooled state. Furthermore, attempts based on this assumption have shown unnatural features.

For inflation, naturality has played an important role. This is understandable since for phenomena that can not be directly observed, one attempts a description starting with the most natural expectations. The importance of naturality principles is to provide guidance from more familiar analogies with the hope of gaining predictability. For inflation we can understand naturality as both a macroscopic and microscopic one. Macroscopically we would like a description that rests with common-day experience. Microscopically it should be consistent with the standard model of particle physics.

Under both categories, the standard scenario has shown unsatisfactory features [2, 3, ,7]. Microscopically slow-roll scalar field dynamics requires an ultra-flat potential, although no such potential is required otherwise for particle phenomenology. Macroscopically, reheating requires globally coherent radiation waves on the scale of the inflated universe. Local incoherent heat transfer is more familiar to experience. Furthermore, a globally coherent heating process requires a large scale radiator, which in the standard scenario is the inflaton. This raises the question about how the random inflaton field configuration before and during inflation attains quantum coherence at the end?

We can accept that naturality principles for inflation do not have rigorous justifications and therefore can be abandoned or softened. However with this, any picture of inflation 
based on familiar analogies would require modifications. This loosens theoretical constraints which otherwise are required to be consistent with only the limited data from observation. As such predictability from theory becomes less definitive. Nevertheless, if that is the way nature works, then that is the way it is.

This would be an acceptable conclusion once all attempts for a natural explanation have been examined. If we digress back to this elementary point, we can rethink about the known ways to induce energy density perturbations. More general to quantum fluctuations are thermal fluctuations. We showed in [8] that in the context of near equilibrium thermodynamics, during inflation thermal fluctuations could dominate quantum fluctuations under certain conditions. Neither energetics nor standard model dynamics precludes a thermal state during inflation. In [9] a model "Warm Inflation" scenario was considered for a self interacting scalar field. The solution had the desirable feature of satisfying observational constraints with minimal specifications placed on the field theory. The condition for slowroll was shifted from requirements on the potential to a frictional force term that coupled the inflaton to a thermalized heat reservoir. By shifting to a frictionally produced slow-roll, it also gave a local heating mechanism.

The first goal of this paper is to clarify the energetics in a thermal inflationary environment. Secondly we derive from quantum field theory a generalized Langevin equation for the scalar inflaton field coupled to a modeled heat reservoir system. In certain limits, which we state, the equation reduces to the one we used in [9]. Finally with these ingredients, an interpretation in terms of particle physics is attempted. To maintain physical clarity, our derivation is Hamiltonian based and performed in a cubic box with periodic boundary conditions. Appendix A provides a convenient reference to thermal properties of free fields that are used often in the text. It also relates our notation to standard form as well as shows how to take the infinite volume limit. Appendix B gives an alternative derivation of the Langevin equation from that in section (III). The purpose for this is discussed in section (III).

\section{ENERGETICS}

Let us account for the total energy in the inflationary universe. Consider a scalar inflaton field with Hamiltonian density defined with respect to the physical volume

$$
\mathcal{H}=\frac{1}{2} \dot{\phi}^{2}+V(\phi)
$$

First suppose that the inflaton is the only system in the universe. If during inflation the inflaton has negligible kinetic energy, $\left\langle\dot{\phi}^{2}\right\rangle \approx 0$, then the energy density during inflation would be all potential from $V\left(\phi_{0}\right)$. Such a situation could occur if $\phi_{0}$ were at a local extrema where $V^{\prime}\left(\phi_{0}\right)=0$. Although the energy density remains constant in this case, the volume $U$ of the universe in physical units would grow after n e-folds to $U(n) \sim R_{0}^{3} e^{3 n}$ where $U(0) \sim R_{0}^{3}$ is the initial volume of the universe just before inflation. In terms of total energy, $E_{T}(n) \equiv U(n)<\mathcal{H}>$, it would be after $n$ e-folds of inflation

$$
E_{T}(n)=V\left(\phi_{0}\right) U(0) e^{3 n}=E_{T}(0) e^{3 n} .
$$


Turning to the more realistic situation in which there is kinetic energy, it is conceptually helpful to first understand energy transfer in the classical limit for the inflaton field. The quantum mechanical problem is treated in section (III). For the classical limit in the expanding universe, the rate of change of the energy density can be expressed as

$$
\frac{d \mathcal{H}(t)}{d t}=-3 H \dot{\phi}^{2}(t)
$$

If in addition the inflaton field expels energy to some other system, one can express this, when treating the inflaton as an isolated system, by adding a dissipative term to the righthand-side of eq. (4). If we choose the specific form to have the lowest time derivative and be even in the field, eq. (4) becomes

$$
\frac{d \mathcal{H}(t)}{d t}=-3 H \dot{\phi}^{2}(t)-\int d t^{\prime} \dot{\phi}(t) \Gamma\left(t, t^{\prime}\right) \dot{\phi}\left(t^{\prime}\right) .
$$

This implies that the equation of motion for the inflaton is

$$
\ddot{\phi}(t)+3 H \dot{\phi}(t)+\int^{t} \Gamma\left(t, t^{\prime}\right) \dot{\phi}\left(t^{\prime}\right) d t^{\prime}+V^{\prime}(\phi(t))=0 .
$$

In the next section we derive a quantum operator equation similar to this but also include a random force term and treat spatial variations.

Having inserted a dissipative term in eqs. (5), energy balance implies that there must be some other system receiving this energy. If the second system is sufficiently large, it will act as a heat reservoir which induces fluctuations on the inflaton field. In the next section we examine a model heat reservoir system which we assume is thermalized. For the reservoir, we do not commit ourselves to a specific particle physics realization. However, in section (IV) a particle physics interpretation is offered. Furthermore, one may question the assumption of thermalization for the heat reservoir. More general would be some other statistical distribution. However for the present work we assume that the heat reservoir is in thermal equilibrium at some temperature T. Further treatment of this problem would require details about the dynamics beyond what we consider. Finally during inflation, the temperature also could be a function of cosmic time, but we consider it fixed.

What is not an assumption and the important point to establish here is the energetic justification of the system - heat reservoir decomposition. The validity of this as well as consistency with the inflation solution requires

$$
\delta \rho_{\phi}(\mathbf{x}, t) \ll \rho_{r}(t) \ll \rho_{\phi}(t) .
$$

Here

$$
\delta \rho_{\phi}(\mathbf{x}, t)=\mathcal{H}(\phi(\mathbf{x}, t))-\mathcal{H}\left(\phi_{0}(t)\right)=V^{\prime}\left(\phi_{0}(t)\right) \delta \phi(\mathbf{x}, t)
$$

is the energy density contained in the fluctuations of the inflaton field

$$
\delta \phi(\mathbf{x}, t)=\phi(\mathbf{x}, t)-\phi_{0}(t),
$$

$\rho_{r}(t)$ is the energy density in the heat reservoir, and $\rho_{\phi}(t)$ is the vacuum energy density, with all of these evaluated at cosmic time t. 
The first requirement, from the right inequality in eq. (7), is the vacuum dominance condition needed for inflation and the second requirement, from the left inequality, is needed for the system-heat reservoir decomposition. In [9] the warm inflation scenarios that we found were for

$$
R \equiv \frac{\rho_{r}}{\rho_{\phi}} \leq 10^{-2} .
$$

This is sufficient to satisfy the first requirement of vacuum energy dominance. This statement can be strengthened for certain solution regimes found, which were consistent with observation and had $R$ one to two orders of magnitude less than eq. (10). The second requirement can be established from the observed amplitude in eq. (1) since [3.10]

$$
10^{-3}-10^{-5} \sim\left(\frac{\delta \rho_{\phi}(\mathbf{k})}{\rho}\right)_{H E}=\left(\frac{\delta \rho_{\phi}(\mathbf{k})}{\dot{\phi}^{2}+\frac{4}{3} \rho_{r}}\right)_{H C} \ll\left(\frac{\delta \rho_{\phi}(\mathbf{k})}{\rho_{\phi}}\right)_{H C} .
$$

Here $H C$ and $H E$ mean horizon crossing and entry respectively, and $\mathbf{k}$ is the comoving wavenumber with horizon crossing occurring at cosmic time $\mathrm{t}$ such that $|\mathbf{k}| e^{-H t} \sim H$. On the left-hand-side $\delta \rho$ from eq. (1) has been equated to $\delta \rho_{\phi}$. In [8] we found that the minimal thermodynamic requirement for a thermal scenario was $\rho_{r} \sim \phi^{2}$, although for the model in [9] for all cases $\rho_{r} \gg \dot{\phi}^{2}$. In either case from eq. (11) we have

$$
\left(\frac{\delta \rho_{\phi}(\mathbf{k})}{\dot{\phi}^{2}+\frac{4}{3} \rho_{r}}\right)_{H C} \sim\left(\frac{\delta \rho_{\phi}(\mathbf{k})}{\rho_{r}}\right)_{H C} \sim 10^{-3}-10^{-5},
$$

which satisfies the second requirement in eq. (7). Thus energetically a thermal component can exist during inflation.

\section{FIELD THEORY}

In this section a field theory derivation of the operator equation of motion for the inflaton is given which has the form of a Langevin-like rate equation. In [8.9] such a rate equation was postulated. Below we consider a simple model heat reservoir system which is coupled linearly to the completely interacting inflaton field. The total system-reservoir Lagrangian is

$$
L_{T}=L_{S}+L_{R}+L_{I}
$$

where on the right-hand-side the Lagrangians are $L_{S}$ for the inflaton (system), $L_{R}$ for the reservoir, and $L_{I}$ for the interaction between system and reservoir. The inflaton's Lagrangian, $L_{S}$, can have an arbitrary potential and accounts for the expansion term. It has the familiar form

$$
L_{S}=\int_{V} d^{3} \mathbf{x} e^{3 H t}\left[\frac{1}{2}\left(\left(\partial_{0} \phi(\mathbf{x}, t)\right)^{2}-\left(e^{-H t} \nabla \phi(\mathbf{x}, t)\right)^{2}-m^{2} \phi^{2}(\mathbf{x}, t)\right)-V(\phi(\mathbf{x}, t))\right],
$$

where the potential can have the general expansion 


$$
V(\phi)=\sum_{n=3}^{\infty} \frac{g_{n}}{n !} \phi^{n}(\mathbf{x}, t)
$$

In this paper we derive the effective operator equation of motion for the inflaton, but we do not study it any further. Thus we will not address the issues of renormalization, which would be connected with solving this equation. As such, our formal derivation is valid whether $L_{S}$ represents an elementary or effective field theory.

The heat reservoir Lagrangian, $L_{R}$, is modeled as a set of free fields each characterized by a mass $\mu_{i}$. It is written as

$$
L_{R}=\sum_{i} \int_{V} d^{3} \mathbf{x} e^{3 H t} \frac{1}{2}\left[\left(\partial_{0} \eta_{i}(\mathbf{x}, t)\right)^{2}-\left(e^{-H t} \nabla \eta_{i}(\mathbf{x}, t)\right)^{2}-\mu_{i}^{2} \eta_{i}^{2}(\mathbf{x}, t)\right] .
$$

Each reservoir field is coupled linearly to the inflaton with a coupling constant $\alpha_{i}$ through the interaction Lagrangian

$$
L_{I}=-\sum_{i} \alpha_{i} \int_{V} d^{3} \mathbf{x} e^{3 H t} \eta_{i}(\mathbf{x}, t) \phi(\mathbf{x}, t)
$$

Note that the coupling constants $\left\{\alpha_{i}\right\}$ carry engineering dimension 2 .

The derivation given below treats the energy transfer between the system and reservoir. However the modeled reservoir in eq. (16) does not have internal interactions. Furthermore energy transfer between the inflaton and the reservoir is not fully treated. To understand the latter two points, note that our problem has a difference to standard problems in Langevin dynamics. The reservoir acts as a large system on the fluctuations of the inflaton field, which is a standard situation for applying Langevin dynamics. However the difference is that the vacuum energy of the inflaton, and particularly through the zero mode, acts as a energy source for maintaining the energy density of the reservoir, which otherwise would diminish due to inflationary expansion. This introduces two complications. The first concerns thermalization for the reservoir. In a standard Langevin problem, it is assumed that the system will weakly interact with the subsystems of the reservoir. There can be many subsystems so that the total effect of the reservoir on the system can be strong. However each subsystem of the reservoir is affected only weakly by the system. In such a circumstance the issue of maintaining an initially thermalized state for the reservoir is not acute. As such, internal reservoir interactions are not crucial to know. In our problem we can accept that the couplings $\alpha_{i}$ in eq. (17) are small (although the derivation below holds for arbitrary $\alpha_{i}$ 's). The question of concern is for whatever vacuum energy that is transferred into the reservoir, can it thermalize on a time scale shorter than $1 / H$ ? Even if our modeled reservoir had internal interactions, to prove thermalization from first principle dynamics would be complicated (some attempts in one-dimensional models are given in [1]).

In our treatment we assume that thermalization occurs and make appropriate by-hand adjustments. These are detailed in the derivation, when relevant. In the next section we will return to the elementary question of thermalization again. Alongside with this problem is the second complication, which is in regards to energy transfer from the inflaton vacuum to the reservoir. Our derivation accounts for only sub-horizon scale physics. As such it treats the fluctuations induced on the modes of the inflaton field by the reservoir, while the modes 
are sub-horizon scale, $\mathbf{k}_{\text {physical }}>H$. However our derivation is not justified for treating the zero mode interaction between the inflaton and the reservoir, since that also involves superhorizon scale physics. The suggestive guess is that the "naive" operator equation derived below for the zero mode, but without a thermalization assumption on the super-horizon scale modes of the reservoir, is approximately valid. We will return to this issue in the conclusion.

It is worth noting that in certain limits the thermal inflation problem reduces to the standard Langevin problem, although this limit did not prove useful in the warm inflation scenarios we considered in [9]. This limit is given in Appendix B as well as the derivation of the Langevin equation in this limit.

Our first goal is to derive the effective equation of motion for the inflaton with the reservoir field variables eliminated. The equation is valid for a time interval $|\Delta t|<H^{-1}$. We will derive the equation for an arbitrary comoving mode of the inflaton field and for the $\mathrm{m}$-th time interval, $t_{m}$ to $t_{m+1}$, with $\mathrm{m}$ arbitrary, where $t_{m} \equiv m \Delta t$ and $t_{0}=0$ is fixed as the starting time of inflation. A complete solution for the inflaton's evolution can be obtained by piecewise construction over all time intervals. The formal derivation is not different for physical wave-numbers that are sub-horizon or super-horizon scale, although as stated above, the approximations leading to the derivation are valid only for the former.

In solving the equations of motion for the reservoir fields, three approximations are made in the $\mathrm{m}$-th time interval for every $\mathrm{m}$. Firstly the red-shifting factor of a given comoving wavenumber $\mathbf{k}_{n}$ is held fixed at $e^{-2 H t_{m}}$. Secondly, at the beginning of every time interval, the state of the reservoir field operators is readjusted. Finally the uncoupled modes of the reservoir fields are assumed to obey a canonical distribution with respect to their physical frequency

$$
\omega_{n\left(t_{m}\right)}^{i} \equiv e^{-2 H t_{m}} \mathbf{k}_{n}^{2}+\mu_{i}^{2} .
$$

Here time $t$ has been demoted into the subscript to signify that it is treated as an adiabatic parameter as far as the wavenumber is concerned.

The first approximation is made to simplify the calculation so that it can be solved analytically. It can be dropped if one is willing to apply more sophisticated methods of solution. The latter two approximations are physically motivated. They are by-hand treatments of the interactions amongst the reservoir fields. Details about the second approximation are given within the derivation when relevant. The third approximation implements our thermalization assumption for the reservoir. In the derivation we are careful to separate these two approximations. The second is made at the operator level. The third is a statement about the state. For it, we are firstly assuming that the description of the reservoir is statistical and secondly that the particular distribution is canonical. In the next section we argue that the reservoir state is created from quantum decay processes, in which case a statistical description is inherently required. The assumption of being a canonical distribution seems the most obvious first guess.

The final equation of motion for the inflaton will be stochastic since the reservoir state is specified by a statistical distribution. The final equation of motion for the inflaton superficially will appear nonconservative since the reservoir fields are going to be eliminated. Under certain conditions placed on the reservoir Hamiltonian, we derive the Langevin equa- 
tion used in [9]. This limit is examined and we verify the fluctuation-dissipation theorem in its standard form [12].

The derivation below follows well known methods from non-equilibrium statistical mechanics which have been refined over the years [13 [16]. A primary motivation for this ongoing effort has been to understand the universal properties of the Langevin equation and to obtain a possible explanation from first principles [13,17]. This believed universality is one reason for us to start our study of stochastic dynamics for thermal scenarios with the Langevin equation. Our derivation below follows closest to [13] and the model for the heat reservoir follows [16]. We have made some modifications to these works, which were for quantum mechanical models, in order that we can treat a quantum field and account for the expansion term. In Appendix B there is an alternative derivation which obtains the rate equation in certain limits for an arbitrary reservoir Hamiltonian.

Before proceeding, Let us review literature that is related to the present work. Applications in cosmology using Langevin dynamics have been done in [18, 19], although both our methods and motivation differ from these works. Studies of finite temperature field theory in Robertson-Walker Universes have been done in [20]. Finally a calculation with similar objectives to ours in this paper is given in [21]. There a path integral derivation is presented of the inflaton's evolution when coupled to a thermal bath in de Sitter space. However the authors did not completely examine the dissipative properties of such a system. As such they apparently missed the connection to warm inflation type scenarios, which for us is the starting motivation to the present formal exercise.

We perform our derivation in a cube for the three spatial directions, which is centered at the origin with sides at $\pm L / 2$ in each direction. The Fourier expansion of a generic field is from eqs. (A9)

$$
\chi(\mathbf{x}, t)=\frac{1}{L^{3}} \sum_{n} \chi\left(\mathbf{k}_{n}, t\right) e^{i \mathbf{k}_{n} \cdot \mathbf{x}},
$$

where throughout this paper we use the notation $\mathbf{k}_{n} \equiv 2 \pi \mathbf{n} / L$, with $\mathbf{n} \equiv\left(n_{x}, n_{y}, n_{z}\right)$ and

$$
\sum_{n} \equiv \sum_{n_{x}=-\infty}^{\infty} \sum_{n_{y}=-\infty}^{\infty} \sum_{n_{z}=-\infty}^{\infty} .
$$

The argument of the coordinate $(\mathbf{x})$ and the momentum $(\mathbf{k})$ space fields are always given to distinguish the two.

The system-reservoir Lagrangian in terms of the Fourier modes as defined in eq. (19) is $L_{T}=L_{S}+L_{R}+L_{I}$, where

$$
\begin{gathered}
L_{S}=\frac{e^{3 H t}}{L^{3}}\left\{\frac{1}{2} \sum_{n}\left[\dot{\phi}\left(\mathbf{k}_{n}, t\right) \dot{\phi}\left(-\mathbf{k}_{n}, t\right)-\left(e^{-2 H t} \mathbf{k}_{n}^{2}+m^{2}\right) \phi\left(\mathbf{k}_{n}, t\right) \phi\left(-\mathbf{k}_{n}, t\right)\right]-V_{F}\right\}, \\
L_{R}=\frac{e^{3 H t}}{L^{3}} \sum_{i} \sum_{n} \frac{1}{2}\left[\dot{\eta}_{i}\left(\mathbf{k}_{n}, t\right) \dot{\eta}_{i}\left(-\mathbf{k}_{n}, t\right)-\left(e^{-2 H t} \mathbf{k}_{n}^{2}+\mu_{i}^{2}\right) \eta_{i}\left(\mathbf{k}_{n}, t\right) \eta_{i}\left(-\mathbf{k}_{n}, t\right)\right]
\end{gathered}
$$

and 


$$
L_{I}=-\frac{e^{3 H t}}{L^{3}} \sum_{i} \sum_{n} \alpha_{i} \eta_{i}\left(\mathbf{k}_{n}, t\right) \phi\left(-\mathbf{k}_{n}, t\right)
$$

with

$$
V_{F} \equiv L^{3} \int d^{3} \mathbf{x} V(\phi)=\sum_{n=3}^{\infty} \frac{1}{L^{3 n-6}} \sum_{m_{1}, \cdots, m_{n-1}} \phi\left(\mathbf{k}_{m_{1}}, t\right) \cdots \phi\left(\mathbf{k}_{m_{n-1}}, t\right) \phi\left(-\mathbf{k}_{m_{1}}-\cdots-\mathbf{k}_{m_{n-1}}, t\right) .
$$

The conjugate momentum to any scalar field $\chi\left(\mathbf{k}_{n}, t\right)$ is

$$
\pi_{\chi}\left(\mathbf{k}_{n}, t\right) \equiv \frac{\partial L}{\partial \dot{\chi}\left(\mathbf{k}_{n}, t\right)}=\frac{e^{3 H t}}{L^{3}} \dot{\chi}\left(-\mathbf{k}_{n}, t\right) .
$$

Converting to the Hamiltonian $H_{T}=\pi_{\phi} \dot{\phi}+\sum_{i} \pi_{\eta_{i}} \dot{\eta}_{i}-L_{T}$, we obtain

$H_{S}=\sum_{n} \frac{1}{2}\left[e^{-3 H t} L^{3} \pi_{\phi}\left(\mathbf{k}_{n}, t\right) \pi_{\phi}\left(-\mathbf{k}_{n}, t\right)+\frac{e^{3 H t}}{L^{3}}\left(e^{-2 H t} \mathbf{k}_{n}^{2}+m^{2}\right) \phi\left(\mathbf{k}_{n}, t\right) \phi\left(-\mathbf{k}_{n}, t\right)\right]+\frac{e^{3 H t}}{L^{3}} V_{F}$,

$$
H_{R}=\sum_{i} \sum_{n} \frac{1}{2}\left[e^{-3 H t} L^{3} \pi_{\eta_{i}}\left(k_{n}, t\right) \pi_{\eta_{i}}\left(-\mathbf{k}_{n}, t\right)+\frac{e^{3 H t}}{L^{3}}\left(e^{-2 H t} \mathbf{k}_{n}^{2}+\mu_{i}^{2}\right) \eta_{i}\left(\mathbf{k}_{n}, t\right) \eta_{i}\left(-\mathbf{k}_{n}, t\right)\right]
$$

and $H_{I}=-L_{I}$. Our notation is that all Hamiltonians have some specifying subscript, which then leaves the Hubble constant to be $H$.

To quantize the theory the postulated equal time commutation relations (CCR) are given in eqs. (A2) and (A11). The operator equations of motion from $H_{T}$ are

$$
\begin{gathered}
\dot{\phi}\left(\mathbf{k}_{n}, t\right)=i\left[H_{T}, \phi\left(\mathbf{k}_{n}, t\right)\right]=e^{-3 H t} L^{3} \pi_{\phi}\left(\mathbf{k}_{n}, t\right) \\
\dot{\pi}_{\phi}\left(\mathbf{k}_{n}, t\right)=i\left[H_{T}, \pi_{\phi}\left(\mathbf{k}_{n}, t\right)\right]=-\frac{e^{3 H t}}{L^{3}}\left[\left(e^{-2 H t} \mathbf{k}_{n}^{2}+m^{2}\right) \phi\left(\mathbf{k}_{n}, t\right)+\frac{\delta V_{F}}{\delta \phi\left(-\mathbf{k}_{n}, t\right)}+\sum_{i} \alpha_{i} \eta_{i}\left(\mathbf{k}_{n}, t\right)\right] \\
\dot{\eta}_{i}\left(\mathbf{k}_{n}, t\right)=e^{-3 H t} L^{3} \pi_{\eta_{i}}\left(\mathbf{k}_{n}, t\right)
\end{gathered}
$$

and

$$
\dot{\pi}_{\eta_{i}}\left(\mathbf{k}_{n}, t\right)=-\frac{e^{3 H t}}{L^{3}}\left[\left(e^{-2 H t} \mathbf{k}_{n}^{2}+\mu_{i}^{2}\right) \eta_{i}\left(\mathbf{k}_{n}, t\right)+\alpha_{i} \phi\left(\mathbf{k}_{n}, t\right)\right]
$$

The resulting second order field equations are

$$
\ddot{\phi}\left(\mathbf{k}_{n}, t\right)+3 H \dot{\phi}\left(\mathbf{k}_{n}, t\right)+\left[e^{-2 H t} \mathbf{k}_{n}^{2}+m^{2}\right] \phi\left(\mathbf{k}_{n}, t\right)+\frac{\delta V_{F}}{\delta \phi\left(-\mathbf{k}_{n}, t\right)}+\sum_{i} \alpha_{i} \eta_{i}\left(\mathbf{k}_{n}, t\right)=0
$$


and

$$
\ddot{\eta}_{i}\left(\mathbf{k}_{n}, t\right)+3 H \dot{\eta}_{i}\left(\mathbf{k}_{n}, t\right)+\left[e^{-2 H t} \mathbf{k}_{n}^{2}+\mu_{i}^{2}\right] \eta_{i}\left(\mathbf{k}_{n}, t\right)+\alpha_{i} \phi\left(\mathbf{k}_{n}, t\right)=0
$$

We now implement our second approximation discussed earlier. At the beginning of every time interval $t_{m}$, we readjust the state of the reservoir fields as

$$
\eta_{i}\left(\mathbf{k}_{n}, t_{m} ; t_{m-1}\right) \rightarrow \eta_{i}^{0}\left(\mathbf{k}_{n}, 0 ; t_{m}\right),
$$

where $\eta_{i}\left(\mathbf{k}_{n}, t ; t_{m-1}\right)$ and $\eta_{i}^{0}\left(\mathbf{k}_{n}, t ; t_{m}\right)$ are the solutions of eq. (33) at time $t$ respectively with and without the coupling term to $\phi$ and with the frequency $\omega_{n(t)}^{i}$ held fixed at respectively $t_{m-1}$ and $t_{m}$. Formally this operation can be viewed as a set of impulsive forces that act on the reservoir. The first purpose of these adjustments is to add sufficient energy so that the reservoir's energy density with respect to physical volume remains constant. Secondly these adjustments are an external treatment of interactions within the reservoir. They shift each field back to its free field state at $t=0$ but with its physical frequency decreased by a little. This operation is in preparation for the thermalization assumption we will make below on the reservoir's state. The first purpose given above acts as a constraint on the inflaton's evolution. Consistency with the inflaton's equation of motion for a particular model was demonstrated in 9]. The discretized treatment simplifies the calculation. One expects the actual dynamics to be smooth and continuous.

The solution for the oscillator field $\eta_{i}$ for the time interval $\left[t_{m}, t_{m+1}\right]$ is

$$
\begin{aligned}
\eta_{i}\left(\mathbf{k}_{n}, t ; t_{m}\right) & =\eta_{i}^{0}\left(\mathbf{k}_{n}, t-t_{m} ; t_{m}\right)-\frac{\alpha_{i}}{\Omega_{n\left(t_{m}\right)}^{i 2}}\left[\phi\left(\mathbf{k}_{n}, t\right)-\phi\left(\mathbf{k}_{n}, t_{m}\right) e^{\frac{-3 H\left(t-t_{m}\right)}{2}} \cos \Omega_{n\left(t_{m}\right)}^{i}\left(t-t_{m}\right)\right. \\
& \left.-e^{-\frac{3}{2} H\left(t-t_{m}\right)} \int_{t_{m}}^{t} d t^{\prime} \cos \Omega_{n\left(t_{m}\right)}^{i}\left(t-t^{\prime}\right) e^{\frac{3}{2} H\left(t^{\prime}-t_{m}\right)}\left(\dot{\phi}\left(\mathbf{k}_{n}, t^{\prime}\right)+\frac{3 H}{2} \phi\left(\mathbf{k}_{n}, t^{\prime}\right)\right)\right]
\end{aligned}
$$

where

$$
\Omega_{n(t)}^{i}=\sqrt{e^{-2 H t} \mathbf{k}_{n}^{2}+\mu_{i}^{2}-\frac{9 H^{2}}{4}}
$$

and

$\eta_{i}^{0}\left(\mathbf{k}_{n}, t ; t_{m}\right)=e^{-\frac{3 H t}{2}}\left[\eta_{i}^{0}\left(\mathbf{k}_{n}, 0\right) \cos \Omega_{n\left(t_{m}\right)}^{i}(t)+\frac{1}{\Omega_{n\left(t_{m}\right)}^{i}}\left(\frac{3 H}{2} \eta_{i}^{0}\left(\mathbf{k}_{n}, 0\right)+L^{3} \pi_{\eta_{i}}\left(\mathbf{k}_{n}, 0\right)\right) \sin \Omega_{n\left(t_{m}\right)}^{i}(t)\right]$

is the solution for the free reservoir fields with the time dependent physical frequency eq. (18) of the comoving mode held fixed at $t_{m}$. As in eq. (18), the subscript $n(t)$ in eq. (36) refers to the physical wavenumber of the comoving mode $\mathbf{k}_{n}$ at cosmic time t. Substituting eq. (35) into eq. (32), we obtain for $t_{m}<t<t_{m+1}$

$$
\ddot{\phi}\left(\mathbf{k}_{n}, t\right)+3 H \dot{\phi}\left(\mathbf{k}_{n}, t\right)+e^{-\frac{3}{2} H\left(t-t_{m}\right)} \sum_{i}\left(\frac{\alpha_{i}}{\Omega_{n\left(t_{m}\right)}^{i}}\right)^{2} \int_{t_{m}}^{t} d t^{\prime} e^{\frac{3}{2} H\left(t^{\prime}-t_{m}\right)} \cos \Omega_{n\left(t_{m}\right)}^{i}\left(t-t^{\prime}\right) \dot{\phi}\left(\mathbf{k}_{n}, t^{\prime}\right)
$$




$$
\begin{aligned}
& +\left(e^{-2 H t} \mathbf{k}_{n}^{2}+m^{2}\right) \phi\left(\mathbf{k}_{n}, t\right)-\sum_{i}\left(\frac{\alpha_{i}}{\Omega_{n\left(t_{m}\right)}^{i}}\right)^{2}\left[\phi\left(\mathbf{k}_{n}, t\right)-\phi\left(\mathbf{k}_{n}, t_{m}\right) e^{-\frac{3}{2} H\left(t-t_{m}\right)} \cos \Omega_{n(t)}^{i}\left(t-t_{m}\right)\right. \\
& \left.-\frac{3 H}{2} e^{-\frac{3}{2} H\left(t-t_{m}\right)} \int_{t_{m}}^{t} d t^{\prime} e^{\frac{3}{2} H\left(t^{\prime}-t_{m}\right)} \cos \Omega_{n(t)}^{i}\left(t-t^{\prime}\right) \phi\left(\mathbf{k}_{n}, t^{\prime}\right)\right] \\
& +\frac{\delta V_{F}(\phi)}{\delta \phi\left(-\mathbf{k}_{n}, t\right)}=\eta\left(\mathbf{k}_{n}, t ; t_{m}\right)
\end{aligned}
$$

where

$$
\eta\left(\mathbf{k}_{n}, t ; t_{m}\right)=-\sum_{i} \alpha_{i} \eta_{i}^{0}\left(\mathbf{k}_{n}, t-t_{m} ; t_{m}\right)
$$

Up to this point, no statistical assumption has been made. Following our earlier discussion, we now assume that the free reservoir fields are canonically distributed. The statistical mechanics for the reservoir system based on our above approximations is the same within each e-fold as in flat-space. For this Appendix A has been provided as a useful reference. As a clarification, no approximation has been made in treating the inflaton system. For the reservoir fields from eq. (A19) for $t_{m}<t, t^{\prime}<t_{m+1}$, we have

$$
\begin{aligned}
\left\langle\left\langle\eta_{i}\left(\mathbf{k}_{n}, t-t_{m} ; t_{m}\right)\right.\right. & \left.\eta_{i}\left(\mathbf{k}_{n}^{\prime}, t^{\prime}-t_{m} ; t_{m}\right\rangle\right\rangle_{T}= \\
& \frac{L^{3}}{2} e^{-\frac{3 H}{2}\left(t+t^{\prime}-2 t_{m}\right)} \delta_{n,-n^{\prime}}\left[\left(\frac{1}{2 \omega_{n\left(t_{m}\right)}^{i}}\left[\cos \omega_{n\left(t_{m}\right)}^{i}\left(t-t^{\prime}\right)+\cos \omega_{n\left(t_{m}\right)}^{i}\left(t+t^{\prime}-2 t_{m}\right)\right]\right.\right. \\
+ & \frac{1}{2 \Omega_{n\left(t_{m}\right)}^{i 2}}\left(\frac{9 H^{2}}{4 \omega_{n\left(t_{m}\right)}^{i}}+\omega_{n\left(t_{m}\right)}^{i}\right)\left[\cos \omega_{n\left(t_{m}\right)}^{i}\left(t-t^{\prime}\right)-\cos \omega_{n\left(t_{m}\right)}^{i}\left(t+t^{\prime}-2 t_{m}\right)\right] \\
+ & \left.\frac{3 H}{2 \Omega_{n\left(t_{m}\right)}^{i} \omega_{n\left(t_{m}\right)}^{i}} \sin \omega_{n\left(t_{m}\right)}^{i}\left(t+t^{\prime}-2 t_{m}\right)\right) \operatorname{coth}\left(\frac{\omega_{n\left(t_{m}\right)}^{i}}{2 T}\right) \\
& \left.-\frac{i}{\Omega_{n\left(t_{m}\right)}^{i}} \sin \omega_{n\left(t_{m}\right)}^{i}\left(t-t^{\prime}\right)\right]
\end{aligned}
$$

Eq. (38) with the assumption eq. (40) is the general rate equation and the main result of this section. For use later, the symmetric correlation function of the force operator is

$$
\begin{aligned}
S_{T}\left(\mathbf{k}_{n}, t, t^{\prime} ; t_{m}\right) & \equiv \frac{1}{2}\left\langle\left\langle\eta\left(\mathbf{k}_{n}, t ; t_{m}\right) \eta\left(-\mathbf{k}_{n}, t^{\prime} ; t_{m}\right)+\eta\left(-\mathbf{k}_{n}, t^{\prime} ; t_{m}\right) \eta\left(\mathbf{k}_{n}, t ; t_{m}\right)\right\rangle\right\rangle_{T} \\
& =\frac{L^{3}}{4} e^{-\frac{3 H}{2}\left(t+t^{\prime}-2 t_{m}\right)} \sum_{i} \alpha_{i}^{2}\left[\frac{1}{\omega_{n\left(t_{m}\right)}^{i}}\left[\cos \omega_{n\left(t_{m}\right)}^{i}\left(t-t^{\prime}\right)+\cos \omega_{n\left(t_{m}\right)}^{i}\left(t+t^{\prime}-2 t_{m}\right)\right]\right. \\
& +\frac{1}{\Omega_{n\left(t_{m}\right)}^{i 2}}\left(\frac{9 H^{2}}{4 \omega_{n\left(t_{m}\right)}^{i}}+\omega_{n\left(t_{m}\right)}^{i}\right)\left[\cos \omega_{n\left(t_{m}\right)}^{i}\left(t-t^{\prime}\right)-\cos \omega_{n\left(t_{m}\right)}^{i}\left(t+t^{\prime}-2 t_{m}\right)\right] \\
& \left.+\frac{3 H}{\Omega_{n\left(t_{m}\right)}^{i} \omega_{n\left(t_{m}\right)}^{i}} \sin \omega_{n\left(t_{m}\right)}^{i}\left(t+t^{\prime}-2 t_{m}\right)\right] \operatorname{coth}\left(\frac{\omega_{n\left(t_{m}\right)}^{i}}{2 T}\right) .
\end{aligned}
$$


We next derive a limiting form of eq. (38). Consider a large number of oscillator fields $\eta_{i}$ that are represented by a continuous distribution as

$$
\sum_{i} \rightarrow \int d \mu N(\mu)
$$

where $N(\mu)$ is the spectral weight function. Assume that $N(\mu)$ is nonzero only in the interval $\mu_{l}<\mu<\mu_{u}$ with $\Delta_{\mu} \equiv \mu_{u}-\mu_{l}$ and the corresponding definition $\Delta_{n\left(t_{m}\right)}^{\Omega} \equiv$ $\sqrt{e^{-2 H t} \mathbf{k}_{n}^{2}+\mu_{u}^{2}-\frac{9 H^{2}}{4}}-\sqrt{e^{-2 H t} \mathbf{k}_{n}^{2}+\mu_{l}^{2}-\frac{9 H^{2}}{4}}$. Also assume that

$$
N(\mu)\left(\frac{\alpha(\mu)}{\Omega_{n\left(t_{m}\right)}^{\mu}}\right)^{2}=\frac{2 \Gamma}{\pi}
$$

is a constant over the interval $\Delta_{\mu}$. If $\Delta_{n\left(t_{m}\right)}^{\Omega} \gg H$, then for $1 / \Delta_{n\left(t_{m}\right)}^{\Omega} \ll t<1 / H$

$$
\int_{0}^{\infty} d \mu N(\mu)\left(\frac{\alpha(\mu)}{\Omega_{n\left(t_{m}\right)}^{\mu}}\right)^{2} \cos \Omega_{n\left(t_{m}\right)}^{\mu} t=2 \Gamma \delta_{1 / \Delta_{n\left(t_{m}\right)}^{\Omega}}(t) .
$$

The subscript on the right-hand-side is to indicate that the $\delta$-function is smeared over a time interval $1 / \Delta_{n\left(t_{m}\right)}^{\Omega}$. Inserting eq. (44) into eq. (38) we obtain

$$
\begin{aligned}
\ddot{\phi}\left(\mathbf{k}_{n}, t\right) & +(3 H+\Gamma) \dot{\phi}\left(\mathbf{k}_{n}, t\right)+\left(e^{-2 H t} \mathbf{k}_{n}^{2}+m^{2}-\frac{2 \Gamma \Delta_{\mu}}{\pi}+\frac{3 \Gamma H}{2}\right) \phi\left(\mathbf{k}_{n}, t\right) \\
& +\frac{\delta V_{F}}{\delta \phi\left(-\mathbf{k}_{n}, t\right)}+2 \Gamma e^{-\frac{3}{2} H\left(t-t_{m}\right)} \phi\left(\mathbf{k}_{n}, t_{m}\right) \delta_{1 / \Delta_{n\left(t_{m}\right)}^{\Omega}}\left(t-t_{m}\right)=\eta^{\prime}\left(\mathbf{k}_{n}, t ; t_{m}\right)
\end{aligned}
$$

where

$$
\eta^{\prime}\left(\mathbf{k}_{n}, t ; t_{m}\right) \equiv-\int d \mu N(\mu) \alpha(\mu) \eta^{0}\left(\mathbf{k}_{n}, t ; t_{m} ; \mu\right)
$$

In the limit of zero expansion, $H=0$, we obtain from eq. 445)

$$
\begin{aligned}
\ddot{\phi}\left(\mathbf{k}_{n}, t\right) & +\Gamma \dot{\phi}\left(\mathbf{k}_{n}, t\right)+\left(\mathbf{k}_{n}^{2}+m^{2}-\frac{2 \Gamma \Delta_{\mu}}{\pi}\right) \phi\left(\mathbf{k}_{n}, t\right) \\
& +\frac{\delta V_{F}}{\delta \phi\left(-\mathbf{k}_{n}, t\right)}+2 \Gamma \phi\left(\mathbf{k}_{n}, t_{m}\right) \delta_{1 / \Delta_{n\left(t_{m}\right)}^{\Omega}}\left(t-t_{m}\right)=\eta_{0}^{\prime}\left(\mathbf{k}_{n}, t\right)
\end{aligned}
$$

where $\eta_{0}^{\prime}\left(\mathbf{k}_{n}, t\right)$ is as in eq. (45) but with $H=0$ in eq. (37). Aside from the last term on the left-hand-side, which is nonzero only for a very short time interval, $1 / \Delta_{\mu}$, this is the standard form of the Langevin equation and the type we used in [9]. In the limit $T \rightarrow \infty$ we find from eq. (41) and eq. (44)

$$
\frac{1}{2}\left\langle\left\langle\eta_{0}\left(\mathbf{k}_{n}, t\right) \eta_{0}\left(-\mathbf{k}_{n}, t^{\prime}\right)+\eta_{0}\left(-\mathbf{k}_{n}, t^{\prime}\right) \eta_{0}\left(\mathbf{k}_{n}, t\right)\right\rangle\right\rangle_{T \rightarrow \infty} \rightarrow 2 L^{3} \Gamma T \delta\left(t-t^{\prime}\right),
$$

which verifies the fluctuation-dissipation theorem [12]. 


\section{INTERPRETATION}

In the last section we obtained the generalized Langevin equation (38), which induces fluctuations on the inflaton as well as frictionally damps its motion. To obtain this equation, we had to introduce a heat reservoir of light particles that interacted with the inflaton. In 99 one form of rate equation (38), namely eq. (47), was shown to be numerically successful for inflaton dynamics. This evidence provides sufficient motivation to seek an interpretation of the heat reservoir in the context of particle physics. The heat reservoir of the last section is a toy model. Here we want to think generally about a second system of light particles which acts as a random force on the inflaton.

For the dynamics described in the last section to be realizable in the real world, in particular two properties are needed for the reservoir. Firstly there must be some mechanism available during inflation to produce the reservoir particles. Secondly these particles must interact rapidly on the scale of the expansion time $1 / H$. In section (III) we assumed that they could thermalize. This stringent of a condition is not needed for eq. (38) to describe stochastic evolution of the inflaton. Nevertheless, we keep with our thermalization assumption and will see how close we can come. In addition to these two properties, a less important point of interest is what direct interpretation the explicit reservoir Hamiltonian (17) in our simple model has.

To address these issues, we first make three general observations. Firstly, the zero mode of the inflaton is evolving quasi-statically during its roll down the potential. Secondly, quantum processes will occur within micro-physical scales, so for physical distances and cosmic time less than $1 / H$ at any e-fold of inflation. The third observation is based on our analysis in [9]. There we found that the energy density of the heat reservoir was close to the GUT scale $M_{\mathrm{GUT}}^{4}$. In particular the temperature range of the thermal scenarios we considered that were consistent with observation was $T \sim(0.01-0.03) M_{\mathrm{GUT}}$. This implies for a single light species a corresponding energy density in the range

$$
\rho_{r} \sim\left(10^{-8}-10^{-6}\right) M_{\mathrm{GUT}}^{4}
$$

For $M_{\mathrm{GUT}}=10^{15} \mathrm{GeV}=1.782 \times 10^{-9} \mathrm{~g}$ and for the lower bound on $\rho_{r}$ this means

$$
\begin{aligned}
\rho_{r} \geq 10^{-8} M_{\mathrm{GUT}}^{4} & =10^{52} \mathrm{GeV}^{4}=10^{66} \mathrm{~g} / \mathrm{cm}^{3} \\
& \approx 10^{51} \rho_{\text {neutron star }} \approx 10^{50} \rho_{\mathrm{HI} \text {-collision }}
\end{aligned}
$$

where $\rho_{\text {neutron star }}$ and $\rho_{\mathrm{HI} \text {-collision }}$ are the energy densities for respectively a neutron star and an upper bound estimate for a heavy-ion collision. For our present purpose, we make the general observation that the energy density of the heat reservoir is large on the scale of the largest probed energy densities.

Observation one provides suitable conditions for particle creation from quantum decay of the inflaton similar to pair-production in a strong quasi-static electric field. This gives a mechanism for producing the particles in the heat reservoir. An outcome is that the description of the reservoir's state must be inherently statistical.

Having made a particle association for the heat reservoir, for completeness of our model in section (III), we make an interpretation of $H_{R}$ in eq. (27). The most direct statement is that $H_{R}$, with an appropriate spectral weight function eq. (42), is a general representation 
of scalar quasiparticle excitations in the noninteracting limit. On the other hand, thinking in terms of elementary fields, if we apply dimensional counting to $H_{I}$ in eq. (17) we find that the reservoir fields $\eta_{i}(\mathbf{x}, t)$ carry engineering dimension 3. This suggests that in our simple model each reservoir field represents a fermion-antifermion pair (ie. $q \bar{q}, e \bar{e}$ etc...), which interacts with the inflaton through a Yukawa coupling. $H_{R}$ then has the interpretation of an effective free field Hamiltonian for paired $f \bar{f}$ states. In QCD these would be the simplest color singlet states constructible from quarks. Beyond these "kinematic" statements, we can not say more at present. Almost nothing is known about matter at the energy densities of eq. (50). We therefore must leave unanswered to what extent $H_{R}$ in eq. (27) and the spectral weight function in eq. (43) approximate a heat reservoir system which can be derived from a realistic particle physics Lagrangian.

Returning from this digression, next we will examine what expectation there is for thermalization in the reservoir. For this let us review the situation up to now. Eq. (38) contains the dynamics that governs energy transfer between the reservoir and the inflaton. The full internal dynamics of the inflaton system in isolation are also treated. Thus eq. (38) is valid for an arbitrary scalar inflaton potential and for reservoir fields that do not interact directly with each other. We discussed in section (III) that in the rapid expansion environment during inflation the internal interactions of an actual reservoir system are essential for distributing any influx of energy. Having established above that the state of the reservoir is inherently statistical, knowledge of internal reservoir dynamics is in particular important for addressing the question of thermalization. In the last section we got around the problem by assuming that thermalization occurs and then made suitable adjustments to our equations.

To check the assumption of thermalization is a difficult problem even if one is given more dynamical details about the reservoir. However based on observations two and three we can get oriented with the scales involved in the problem. For this let us consider an unjustifiable classical model of light hard rocks of average energy $E$, center-of-mass two-body elastic cross section $\sigma$, and at a energy density $\rho_{\text {rocks }}$ of order eq. (50). The mean free path in this system is

$$
l_{\text {rocks }}=\frac{E}{\rho_{\text {rocks }} \sigma},
$$

which for relativistic rocks implies a collision frequency $\omega_{\text {rocks }}=1 / l_{\text {rocks }}$.

We assume that the elastic cross section is independent of energy. Up to logarithmic corrections, this corresponds to Regge asymptotic behavior from single pomeron exchange with slope one (for a review please see [22 and references therein). For a conservative lower bound to elastic $p p$-scattering at high energy, we use $\sigma_{e l}=10 \mathrm{GeV}^{-2}$. The energy of a typical light particle at temperature $T$ is $E \sim T$. Taking the lower bound from eq. (50) $\rho_{\text {rocks }}=10^{52} \mathrm{GeV}^{4}$ which corresponds to $T=0.01 M_{\mathrm{GUT}}$, we find

$$
\omega_{\text {rocks }}=10^{40} \mathrm{GeV} \approx 10^{30} H .
$$

It is known that most of the elastic cross section $\sigma_{e l}$ is increasingly in the forward direction with increasing energy. Accounting for this, we take the average momentum transfer to be $|t|=0.025 \mathrm{GeV}^{2}$. Let us assume statistical independence between collisions for a typical light particle. Then within one e-fold, the root-mean-square energy that the particle transfers 
will be about an order of magnitude greater than its total energy and it samples all angular directions in phase space. Optimistically the range for eq. (52) could be increased by a factor $10^{3}$ by using the upper bound on $\rho_{\text {rocks }}$ in eq. (49) and increasing $\sigma_{e l}$ by over a factor 10. This range presents a case that some sort of GUT-plasma could thermalize within an e-fold at GUT-scaled energy densities.

\section{MECHANICAL ANALOGUE}

In section (III) we derived a quantum Langevin equation, but we can think of it as a classical Langevin equation. For this we can make a picture of the dynamics for a classical inflaton field $\phi^{c}(\mathbf{x}, t)$. We define the order parameter in the classical system as

$$
\phi^{c}(t) \equiv \int_{V} \frac{d^{3} \mathbf{x}}{V} \phi^{c}(\mathbf{x}, t)
$$

and it will sometimes be denoted as $\phi^{c}$. For the classical theory we will make a mechanical model and then associate it with the classical field. We will work in the infinite volume limit where correspondence between the classical and quantum fields can be stated more simply.

Let us first recall some details about the inflaton Hamiltonian. In thermal scenarios the symmetry breaking potential $V(\phi)$ can be of the standard double-well type without an ultraflat region as demonstrated in 99. The relevant magnitudes which define the potential are the same as in the standard scenario. Thus the vacuum energy $V(0) \sim M_{\text {GUT }}^{4}$, the minima of the wells are at $\phi_{m} \approx \pm M_{\mathrm{GUT}}$, and the curvature at the minima are $V^{\prime \prime}\left(\phi_{m}\right) \approx M_{\mathrm{GUT}}^{2}$. Inflation begins when the inflaton tunnels out of the meta-stable minima at $\phi=0$ to $0<\phi_{i}<\phi_{m}$. We will define this moment as the origin of cosmic time $t$. Our classical analogy begins at $\mathrm{t}=0$.

With these preliminary remarks, let us state our classical analog model. We picture the classical scalar field as composed of heavy particles of mass $M_{\phi}=\sqrt{V^{\prime \prime}\left(\phi_{m}\right)}$. They sit in a cubic lattice arrangement with massless (anharmonic if preferred) springs connecting them. Kinetic energy is associated with the particle masses and their motional energy. Potential energy is associated with the energy in the massless springs. The order parameter $\phi^{c}(t)$ is interpreted to be related to the number density $n_{\phi}(t)$ of $\phi$-particles. To obtain a precise relationship we will equate the order parameter with the vacuum expectation value of the quantum Heisenberg scalar field at $\mathrm{t}=0$ as

$$
\phi^{c}(0)=\lim _{V \rightarrow \infty}\left\langle 0\left|\int_{V} \frac{d^{3} \mathbf{x}}{V} \phi(\mathbf{x}, 0)\right| 0\right\rangle
$$

We will assume that the vacuum is a coherent state of zero momentum particles. Using the number density operator

$$
\hat{n} \equiv \lim _{V \rightarrow \infty} \frac{\hat{N}}{V}=\langle\rangle-\lim _{V \rightarrow \infty} \frac{1}{V} \sum_{n} a_{n}^{\dagger} a_{n},
$$

we arrive at 


$$
\phi^{c}(0)=\sqrt{\frac{2 n_{\phi}(0)}{M_{\phi}}} .
$$

In eq. (55) \langle\rangle$-\lim$ means evaluate the operator matrix element in finite volume and then take the limit $V \rightarrow \infty$. In our classical model we define the order parameter at all time with this relation,

$$
\phi^{c}(t) \equiv \sqrt{\frac{2 n_{\phi}(t)}{M_{\phi}}} .
$$

If one prefers, this can be regarded as an unmotivated definition, which relates the the classical mechanical model with our classical field model.

The initial conditions at $t=0$ are the order parameter is at $\phi^{c}(0)=\phi_{i}$, the masses are at rest with number density given by eq. (57), and the springs are elongated such that the cubic symmetry is maintained but the fundamental cell is bigger. When the system is released a breathing mode commences, in which the fundamental cell contracts to a minima and the expands back to its original size at $t=0$ and so on. Correspondingly, the number density oscillates.

To this system, we will add many light $\eta$-particles of mass $m_{\eta} \ll M_{\phi}$ that are thermalized at temperature $\mathrm{T}$. This system forms the heat reservoir. The $\eta$-particles randomly will impart momentum on the $\phi$-particles. This will perturb the breathing mode of the $\phi$-particles as well as excite transverse modes on the $\phi$-lattice. The system so described is similar to that for Brownian motion, except now there are several Brownian particles and they also have an interaction amongst themselves. With minor changes, one can understand the rest of the statistical mechanics for our system from Brownian motion. Slow-roll motion in our classical model corresponds to over-damping, in which the fundamental cell slowly contracts to equilibrium with no oscillations. In the process the potential energy in the $\phi$-system is transferred to the heat reservoir. One can also expand this picture to include inflationary expansion and $\eta$-particle creation, but we will leave that for the reader's thoughts.

\section{CONCLUSION}

In section (III) we obtained the rate eq. (38) which describes the dynamics of the inflaton's comoving modes while they are sub-horizon size. For the zero mode, eq. (38) is therefore unjustified, since it does not treat super-horizon scale physics. What one needs is an equation that describes the transfer of potential energy from the inflaton's vacuum during its roll down to the potential minima. In our model, the energy is transfered not only into the inflaton's kinetic energy, but also into the fields of the reservoir.

In a local patch and for a short time interval, eqs. (38) would be valid for all modes which are ad-hocally defined with respect to the patch. A network of such patches could be constructed to cover the inflating universe for some chosen cosmic time interval. In each patch the respective Langevin equation could be solved with solutions matched at the boundaries. To compute the initial sub-horizon scale energy density perturbations, recall that they are determined by the sub-horizon scale modes. Therefore this computation 
could be done from the solution of the Langevin equation from any one patch. For the zero mode, there are two points of interest. First we want to know the global energy loss by the inflaton. Due to the lack of coherence between patches, the expectation value of the inflaton (order parameter) need not be the same in different patches. Different local regions of the universe could then be in different stages of inflation, depending on the local order parameter. For computing the total change in vacuum energy, one could sum the result from the independent patches. However turning to the second point, for inflation to solve the smoothness problem, one requires the order parameter to be approximately globally homogeneous. One may argue that the local conditions in all patches would be similar, so that the order parameter should not differ by too much in different patches. To verify this would require further investigation within specific models. In particular, to meet observational constraints, one wants to know the degree of homogeneity of the order parameter within an inflating region for a duration of at least 70 e-folds. All statements so far apply equally well to scenarios based on both quantum and thermal fluctuations. There is one difference. Thermal scenarios, do not require the quantum state of the inflaton's zero mode to be coherent over the entire inflationary patch. This is required in the standard scenario for reheating.

The derivation in section (III) is formally valid for a fundamental or effective inflaton Lagrangian $L_{S}$ in eq. (14). Given an initial fundamental Lagrangian for the inflaton, one anticipates that to formulate a consistent stochastic process, it will require high frequency interactions of the inflaton with itself and with other fields to be treated first and appear in the form of an effective Lagrangian $L_{S}$ in eq. (14). We have not developed in this paper any methods for obtaining the appropriate effective Lagrangian one should use for $L_{S}$.

In [9] we assumed near equilibrium dynamics applied everywhere. We therefore used the finite temperature effective potential in $L_{S}$. However nonequilibrium considerations such as done in [23] for flat space may be important to extend for the inflationary era of expanding space. Generally for a scalar inflaton field coupled to gauge fields, such as in GUT theories, one should expect quantum corrections from self-coupling and quantum plus thermal corrections from coupling to the gauge fields to give a modified potential in $L_{S}$. Since the relevant temperature scale for slow-roll dynamics is of order or less than the inflaton mass, one does not expect thermal corrections from the inflaton's self-coupling to be substantial.

To summarize, in this paper we showed that the presence of a sizable thermal component during inflation is energetically allowed. Following our hypothesis in [8], we treated the thermal component as a heat reservoir, which interacted with the inflaton in the manner of Langevin dynamics. We demonstrated in section (III) that the Langevin equation postulated in [8] could be derived from quantum field theory. In [9] we showed that such a dynamical arrangement, for the specific case of a double-well scalar potential, was numerically successful in describing observational data without fine tuning the coupling constant.

One of the improvements suggested by our calculations there was to have a time dependent temperature that smoothly varied by a factor 2-3 over the course of the roll-down period. Not only was this numerically preferable, but it seems that thermodynamics does not restrict some variation in the temperature just before to just after inflation. As such the general dynamical equations during inflation should be able to accommodate such a variation, although specific models may make specific predictions. 
In [9] the reservoir was defined by its thermodynamic properties, but was not given a dynamical representation. Thus we had no underlying mechanism that could explain temperature variations. In section (III) our model supplies an explanation. However it is clear from our treatment there, that it was an arbitrary decision to hold the reservoir's energy density constant with respect to physical volume all throughout inflation. Just as well within the same dynamical framework, it could increase or decrease and in different time periods do either. However this is as far as the model in its present form can go. On the plus side, the model goes far enough to dynamically explain a time dependent reservoir energy density. However the model is not sufficiently constrained to do better than this. The options to improve this situation are to determine a further constraint from either formal means or phenomenological motivation.

\section{ACKNOWLEDGMENTS}

I thank J. Pullin, L. Z. Fang and A. Ashtekar for helpful discussions. This work was supported by the U.S. Department of energy under grant numbers DE-FG02-90ER-40577 and DE-FG02-93ER40771.

\section{APPENDIX A: STATISTICAL MECHANICS OF FREE FIELDS}

Some statistical mechanical properties of free field, which are often used in the text, are given here. We consider a free field Hamiltonian defined in a box of volume $V_{L}$ centered at the origin with sides at $\pm L / 2$ in all three spatial directions. We give results in the discrete form in $V_{L}$ for finite but large $L$ and then in the infinite volume limit, $V_{\infty}$, where $L \rightarrow \infty$. The free field Hamiltonian which we study is

$$
H=\int_{V_{L}} d^{3} \mathbf{x} \frac{1}{2}\left[\pi_{\chi}^{2}(\mathbf{x}, t)+(\nabla \chi(\mathbf{x}, t))^{2}+m^{2} \chi^{2}(\mathbf{x}, t)\right]+C,
$$

where $\mathrm{C}$ is a constant that later will be set so that the lowest energy state is at zero. The fields satisfy the equal time canonical commutation relations (CCR)

$$
\begin{aligned}
{\left[\chi(\mathbf{x}, t), \chi\left(\mathbf{x}^{\prime}, t\right)\right] } & =0 \\
{\left[\pi_{\chi}(\mathbf{x}, t), \pi_{\chi}\left(\mathbf{x}^{\prime}, t\right)\right] } & =0 \\
{\left[\pi_{\chi}(\mathbf{x}, t), \chi\left(\mathbf{x}^{\prime}, t\right)\right] } & =-i \delta^{(3)}\left(\mathbf{x}-\mathbf{x}^{\prime}\right) .
\end{aligned}
$$

The field have the expansion in terms of creation and annihilation operators

$$
\begin{aligned}
\chi(\mathbf{x}, t) & =\frac{1}{L^{3 / 2}} \sum_{n_{x}, n_{y}, n_{z}=-\infty}^{\infty} \frac{1}{\sqrt{2 \omega_{n}}}\left[a_{n} e^{i\left(\mathbf{k}_{n} \cdot \mathbf{x}-\omega_{n} t\right)}+a_{n}^{\dagger} e^{-i\left(\mathbf{k}_{n} \cdot \mathbf{x}-\omega_{n} t\right)}\right] \\
\pi_{\chi}(x, t) & =\frac{-i}{L^{3 / 2}} \sum_{n_{x}, n_{y}, n_{z}=-\infty}^{\infty} \sqrt{\frac{\omega_{n}}{2}}\left[a_{n} e^{i\left(\mathbf{k}_{n} \cdot \mathbf{x}-\omega_{n} t\right)}-a_{n}^{\dagger} e^{-i\left(\mathbf{k}_{n} \cdot \mathbf{x}-\omega_{n} t\right)}\right]
\end{aligned}
$$

where 


$$
\begin{gathered}
\mathbf{k}_{n} \equiv \frac{2 \pi \mathbf{n}}{L}, \\
\mathbf{n} \equiv\left(n_{x}, n_{y}, n_{z}\right), \\
\omega_{n} \equiv \sqrt{\mathbf{k}_{n}^{2}+m^{2}},
\end{gathered}
$$

and from eq. (A2)

$$
\begin{gathered}
{\left[a_{n}, a_{n^{\prime}}\right]=\left[a_{n}^{\dagger}, a_{n^{\prime}}^{\dagger}\right]=0} \\
{\left[a_{n}, a_{n^{\prime}}^{\dagger}\right]=\delta_{n n^{\prime}} .}
\end{gathered}
$$

We define the Fourier transform of the fields as

$$
\begin{gathered}
\chi\left(\mathbf{k}_{n}, t\right) \equiv \int_{V_{L}} d^{3} \mathbf{x} \chi(\mathbf{x}, t) e^{-i \mathbf{k}_{n} \cdot \mathbf{x}} \\
\pi_{\chi}\left(\mathbf{k}_{n}, t\right) \equiv \frac{1}{L^{3}} \int_{V_{L}} d^{3} \mathbf{x} \pi(\mathbf{x}, t) e^{-i \mathbf{k}_{n} \cdot \mathbf{x}} .
\end{gathered}
$$

From eq. (A2) one can verify that the Fourier space modes of the fields satisfy the equal time CCR

$$
\begin{aligned}
{\left[\chi\left(\mathbf{k}_{n}, t\right), \chi\left(\mathbf{k}_{n^{\prime}}, t\right)\right] } & =0 \\
{\left[\pi_{\chi}\left(\mathbf{k}_{n}, t\right), \pi_{\chi}\left(\mathbf{k}_{n^{\prime}}, t\right)\right] } & =0 \\
{\left[\pi_{\chi}\left(\mathbf{k}_{n}, t\right), \chi\left(-\mathbf{k}_{n^{\prime}}, t\right)\right] } & =-i \delta_{n n^{\prime}} .
\end{aligned}
$$

The expansion of the Fourier-modes in terms of the creation-annihilation operators is

$$
\begin{aligned}
\chi\left(\mathbf{k}_{n}, t\right) & =\frac{L^{3 / 2}}{\sqrt{2 \omega_{n}}}\left[a_{n} e^{-i \omega_{n} t}+a_{-n}^{\dagger} e^{i \omega_{n} t}\right] \\
\pi_{\chi}\left(\mathbf{k}_{n}, t\right) & =\frac{-i}{L^{3 / 2}} \sqrt{\frac{\omega_{n}}{2}}\left[a_{n} e^{-i \omega_{n} t}-a_{-n}^{\dagger} e^{i \omega_{n} t}\right] .
\end{aligned}
$$

The constant $\mathrm{C}$ in eq. (A1) is be defined as

$$
C=-\frac{1}{2} \sum_{n} \omega_{n}
$$

Two equivalent forms of the Hamiltonian $\mathrm{H}$ in eq. (A1) are given below. Writing

$$
H=\sum_{n} H_{n}=\sum_{n} H_{k_{n}}
$$

where in terms of the creation-annihilation operators in eq. (A8), 


$$
H_{n}=\omega_{n} a_{n}^{\dagger} a_{n}
$$

and in terms of the Fourier-modes in eq. (A12)

$$
H_{k_{n}}=\frac{L^{3}}{2} \pi_{\chi}\left(\mathbf{k}_{n}, t\right) \pi_{\chi}\left(-\mathbf{k}_{n}, t\right)+\frac{1}{2 L^{3}}\left(\mathbf{k}_{n}^{2}+m^{2}\right) \chi\left(\mathbf{k}_{n}, t\right) \chi\left(-\mathbf{k}_{n}, t\right) .
$$

The partition function, $Z \equiv \operatorname{Tr}\left(e^{-H / T}\right)$, evaluated in the creation-annihilation basis is a product $Z=\prod_{n} Z_{n}$, where

$$
Z_{n}=\operatorname{Tr}\left(e^{-H_{n} / T}\right)=\frac{1}{1-e^{-\omega_{n} / T}} .
$$

The two-point thermal correlation function for the creation-annihilation operators is

$$
\begin{aligned}
\frac{1}{Z_{n}} \operatorname{Tr}\left(e^{\frac{-H_{n}}{T}} a_{n}^{\dagger} a_{n^{\prime}}\right) & \equiv\left\langle\left\langle a_{n}^{\dagger} a_{n^{\prime}}\right\rangle\right\rangle_{T}=\delta_{n n^{\prime}} \frac{1}{e^{\omega_{n} / T}-1} \\
\left\langle\left\langle a_{n} a_{n^{\prime}}\right\rangle\right\rangle_{T} & =\left\langle\left\langle a_{n}^{\dagger} a_{n^{\prime}}^{\dagger}\right\rangle\right\rangle_{T}=0
\end{aligned}
$$

and for the Fourier-modes is

$$
\begin{aligned}
\frac{1}{Z} \operatorname{Tr}\left(e^{\frac{-H}{T}} \chi\left(\mathbf{k}_{n}, t\right) \chi\left(\mathbf{k}_{n}^{\prime}, t^{\prime}\right)\right) & \equiv\left\langle\left\langle\chi\left(\mathbf{k}_{n}, t\right) \chi\left(\mathbf{k}_{n}^{\prime}, t^{\prime}\right\rangle\right\rangle_{T}\right. \\
& =\delta_{n,-n^{\prime}} \frac{L^{3}}{2 \omega_{n}}\left[\operatorname{coth}\left(\frac{\omega_{n}}{2 T}\right) \cos \omega_{n}\left(t-t^{\prime}\right)-i \sin \omega_{n}\left(t-t^{\prime}\right)\right] \\
\left\langle\left\langle\pi_{\chi}\left(\mathbf{k}_{n}, t\right) \pi_{\chi}\left(\mathbf{k}_{n}^{\prime}, t^{\prime}\right)\right\rangle\right\rangle_{T} & =\delta_{n,-n^{\prime}} \frac{\omega_{n}}{2 L^{3}}\left[\operatorname{coth}\left(\frac{\omega_{n}}{2 T}\right) \cos \omega_{n}\left(t-t^{\prime}\right)-i \sin \omega_{n}\left(t-t^{\prime}\right)\right] \\
\left\langle\left\langle\chi\left(\mathbf{k}_{n}, t\right) \pi_{\chi}\left(\mathbf{k}_{n}^{\prime}, t^{\prime}\right)\right\rangle\right\rangle_{T} & =\delta_{n,-n^{\prime}} \frac{1}{2}\left[\operatorname{coth}\left(\frac{\omega_{n}}{2 T}\right) \sin \omega_{n}\left(t-t^{\prime}\right)+i \cos \omega_{n}\left(t-t^{\prime}\right)\right] .
\end{aligned}
$$

From the last relation note that

$$
\left\langle\left\langle\left[\pi_{\chi}\left(\mathbf{k}_{n}, t\right), \chi\left(\mathbf{k}_{n}^{\prime}, t^{\prime}\right)\right]\right\rangle\right\rangle_{T}=-i \delta_{n,-n^{\prime}} \cos \omega_{n}\left(t-t^{\prime}\right) .
$$

The total ensemble averaged energy of the thermalized system is

$$
U_{V_{L}}(T)=\sum_{n} U_{V_{L}}^{n}(T)
$$

where

$$
U_{V_{L}}^{n}(T)=\omega_{n}\left\langle\left\langle a_{n}^{\dagger} a_{n}\right\rangle\right\rangle_{T}=\frac{\omega_{n}}{e^{\omega_{n} / T}-1} .
$$

Let us examine the infinite volume limit. The creation and annihilation operators must be rescaled as

$$
c\left(\mathbf{k}_{n}\right) \equiv\left(\frac{L}{2 \pi}\right)^{3 / 2} a_{n}
$$

and similarly for $a_{n}^{\dagger}$. Substituting eq. (A23) into eq. (A3) and identifying 


$$
\left(\frac{2 \pi}{L}\right)^{3}=\left(\frac{2 \pi \Delta n}{L}\right)_{L \rightarrow \infty}^{3} \rightarrow d^{3} \mathbf{k}
$$

we get the continuum form

$$
\chi(\mathbf{x}, t)=\int \frac{d^{3} \mathbf{k}}{\left[(2 \pi)^{3} 2 \omega_{\mathbf{k}}\right]^{1 / 2}}\left[c(\mathbf{k}) e^{i\left(\mathbf{k}_{n} \cdot x-\omega_{\mathbf{k}} t\right)}+c^{\dagger}(\mathbf{k}) e^{\left.-i\left(\mathbf{k}_{n} \cdot x-\omega_{\mathbf{k}}\right) t\right)}\right]
$$

and similarly for $\pi_{\chi}(\mathbf{x}, t)$. For the momentum space Fourier transforms of the fields in the infinite volume limit, $V_{L}$ must be replaced by $V_{\infty}$ in eqs. (A9) and (A10) and $\pi_{\chi}(\mathbf{k}, t)$ must be defined without the factor $1 / L^{3}$. The Hamiltonian in the forms eq. (A15) and eq. (A16) in $V_{\infty}$ are respectively

$$
\begin{aligned}
H & =\int d^{3} \mathbf{k} \omega_{\mathbf{k}} c^{\dagger}(\mathbf{k}) c(\mathbf{k}) \\
& =\int \frac{d^{3} \mathbf{k}}{(2 \pi)^{3}} \frac{1}{2}\left[\pi_{\chi}(\mathbf{k}, t) \pi_{\chi}(-\mathbf{k}, t)+\left(\mathbf{k}^{2}+m^{2}\right) \chi(\mathbf{k}, t) \chi(-\mathbf{k}, t)\right] .
\end{aligned}
$$

The energy density in $V_{\infty}$ is obtained as

$$
u(T)=\lim _{L \rightarrow \infty} \frac{U_{V_{L}}(T)}{L^{3}} .
$$

From eq. (A21) and (A22) we find

$$
u(T)=\int \frac{d^{3} \mathbf{k}}{(2 \pi)^{3}} \frac{\omega_{\mathbf{k}}}{e^{\omega_{\mathbf{k}} / T}-1} .
$$

The familiar formula for the energy density of electromagnetic radiation is twice this, due to two polarization states for the photon, and with $m=0$ so that $\omega_{\mathbf{k}}=|\mathbf{k}|$.

\section{APPENDIX B: ALTERNATIVE DERIVATION OF THE RATE EQUATION}

For a system with a nearly harmonic potential, such as within one well of a double-well potential, one can derive the Langevin equation with a general reservoir Hamiltonian $H_{R}$ in the regime

$$
H \ll \Gamma \ll \omega
$$

where $H$ is the Hubble constant, $\Gamma$ is the dissipative constant and $\omega$ is the frequency of the system with no anharmonic terms in the potential. We perform the derivation in this appendix. It follows the method of [14. As in section III, it is done in a cube for the three spatial directions, which is centered at the origin with sides at $\pm L / 2$ in each direction.

The Hamiltonian we examine is

$$
H_{T}=H_{S}+H_{R}+H_{I}
$$

where $H_{S}$ is the system Hamiltonian given for the inflaton field in eq. (26), $H_{R}$ is the Hamiltonian for the reservoir system when it is uncoupled to the scalar field system, and 


$$
H_{I}=\alpha \int_{V} d^{3} \mathbf{x} e^{3 H t} \Omega(\mathbf{x}, t) \phi(\mathbf{x}, t)
$$

where $\Omega(\mathbf{x}, t)$ is a Hermitian operator made of any field or composite fields of the reservoir system that couples to the scalar field. For our derivation, we do not need specific details about $H_{R}$. It may well represent a strongly interacting system with respect to $\Omega(\mathbf{x}, t)$. Expressed in momentum space, eq. (B3) is

$$
H_{I}=\frac{e^{3 H t}}{L^{3}} \sum_{n} \alpha \Omega\left(\mathbf{k}_{n}, t\right) \phi\left(-\mathbf{k}_{n}, t\right) .
$$

Postulating the standard equal time CCR of eqs. (A2) and (A11), the equations of motion for the inflaton are

$$
\begin{gathered}
\dot{\phi}\left(\mathbf{k}_{n}, t\right)=i\left[H_{T}, \phi\left(\mathbf{k}_{n}, t\right)\right]=e^{-3 H t} L^{3} \pi_{\phi}\left(\mathbf{k}_{n}, t\right) \\
\dot{\pi}_{\phi}\left(\mathbf{k}_{n}, t\right)=i\left[H, \pi_{\phi}\left(\mathbf{k}_{n}, t\right)\right]=-\frac{e^{3 H t}}{L^{3}}\left[\omega_{n}^{2}(t) \phi\left(\mathbf{k}_{n}, t\right)+\frac{\delta V_{F}}{\delta \phi\left(-\mathbf{k}_{n}, t\right)}+\alpha \Omega\left(\mathbf{k}_{n}, t\right)\right]
\end{gathered}
$$

where

$$
\omega_{n}(t)=\sqrt{e^{-2 H t} \mathbf{k}_{n}^{2}+m^{2}} .
$$

This implies the second order equation of motion

$$
\ddot{\phi}\left(\mathbf{k}_{n}, t\right)+3 H \dot{\phi}\left(\mathbf{k}_{n}, t\right)+\omega_{n}^{2}(t) \phi\left(\mathbf{k}_{n}, t\right)+\frac{\delta V_{F}}{\delta \phi\left(-\mathbf{k}_{n}, t\right)}+\alpha \Omega\left(\mathbf{k}_{n}, t\right)=0 .
$$

We derive the effective equation of motion for a given comoving mode of the inflaton. In the parametric regime eq. (B1), the time interval $1 / \Gamma \ll \Delta t \ll 1 / H$ is sufficient to study the relaxational dynamics of the inflaton. Thus we can drop the second term in eq. (B8) above and assume that the comoving wavenumber $\mathbf{k}_{n}$ is constant at $e^{-2 H t} \mathbf{k}_{n}=\mathbf{k}_{n}^{m}$, where $\mathbf{k}_{n}^{m}$ is the initial value in the time interval $m$ of interest. Dropping the superscript $m$, the resulting second order equation of motion is

$$
\ddot{\phi}\left(\mathbf{k}_{n}, t\right)+\omega_{n}^{2} \phi\left(\mathbf{k}_{n}, t\right)+\frac{\delta V_{F}}{\delta \phi\left(-\mathbf{k}_{n}, t\right)}+\alpha \Omega\left(\mathbf{k}_{n}, t\right)=0
$$

where

$$
\omega_{n}=\sqrt{\mathbf{k}_{n}^{2}+m^{2}}
$$

This can be obtained from the first order equations in eqs. (B5) and (B6) by the replacement

$$
e^{-2 H t} \mathbf{k}_{n}^{2} \rightarrow e^{-2 H t_{m}} \mathbf{k}_{n}^{2}
$$

in $\omega_{n}(t)$ in eq. (B6) and by setting $H=0$ everywhere else.

Doing this, the solution to eqs. (B5) and (B6) are respectively 


$$
\begin{aligned}
\phi\left(\mathbf{k}_{n}, t\right) & =\phi_{0}\left(\mathbf{k}_{n}, t\right)-\frac{\alpha}{\omega_{n}} \int_{0}^{t} d t^{\prime} \Omega\left(\mathbf{k}_{n}, t^{\prime}\right) \sin \omega_{n}\left(t-t^{\prime}\right) \\
& -\frac{1}{\omega_{n}} \int_{0}^{t} d t^{\prime} \frac{\delta V(\phi)}{\delta \phi\left(-\mathbf{k}_{n}, t^{\prime}\right)} \sin \omega_{n}\left(t-t^{\prime}\right) \\
\pi\left(\mathbf{k}_{n}, t\right) & =\pi_{0}\left(\mathbf{k}_{n}, t\right)-\frac{\alpha}{\omega_{n}} \int_{0}^{t} d t^{\prime} \Omega\left(\mathbf{k}_{n}, t^{\prime}\right) \cos \omega_{n}\left(t-t^{\prime}\right) \\
& -\frac{1}{\omega_{n}} \int_{0}^{t} d t^{\prime} \frac{\delta V(\phi)}{\delta \phi\left(-\mathbf{k}_{n}, t^{\prime}\right)} \cos \omega_{n}\left(t-t^{\prime}\right),
\end{aligned}
$$

where $\phi_{0}$ and $\pi_{0}$ are the solutions of eqs. (B5) and (B6) with $V_{F}=0$ and $\alpha=0$. Again for notational convenience we denote the physical momentum simply by $\mathbf{k}_{n}$. The associated comoving mode can be computed at time $t_{m}$ from eq. (B11).

The inflaton (system) can be treated as a small perturbation on $\Omega\left(\mathbf{k}_{n}, t\right)$, however full account must be taken of the heat reservoir's effect on the inflaton. Thus we will account for the lowest order effect of the system on the reservoir coordinate $\Omega\left(\mathbf{k}_{n}, t\right)$, which gives

$$
\begin{aligned}
\Omega\left(\mathbf{k}_{n}, t\right) & =e^{-i H t} \Omega\left(\mathbf{k}_{n}, 0\right) e^{i H t} \\
& =\Omega_{0}\left(\mathbf{k}_{n}, t\right)+\frac{i \alpha}{L^{3}}\left\{\Omega_{0}\left(\mathbf{k}_{n}, t\right) \sum_{n^{\prime}} \int_{0}^{t} d t^{\prime} \phi\left(\mathbf{k}_{n^{\prime}}, t^{\prime}\right) \Omega_{0}\left(-\mathbf{k}_{n^{\prime}}, t^{\prime}\right)\right. \\
& \left.-\sum_{n^{\prime}} \int_{0}^{t} d t^{\prime} \phi\left(\mathbf{k}_{n^{\prime}}, t-t^{\prime}\right) \Omega_{0}\left(-\mathbf{k}_{n}, t-t^{\prime}\right) \Omega_{0}\left(\mathbf{k}_{n}, t\right)\right\}
\end{aligned}
$$

where

$$
\Omega_{0}\left(\mathbf{k}_{n}, t\right)=e^{-i H_{R} t} \Omega\left(\mathbf{k}_{n}, 0\right) e^{i H_{R} t}
$$

is the time development of $\Omega$ in the uncoupled reservoir.

Substituting this into the second term on the right-hand-side of eq. (B12), this term becomes

$$
\frac{\alpha}{\omega_{n}} \int_{0}^{t} d t^{\prime} \Omega\left(\mathbf{k}_{n}, t^{\prime}\right) \sin \omega_{n}\left(t-t^{\prime}\right)=\frac{\alpha}{\omega_{n}} \int_{0}^{t} d t^{\prime} \Omega_{0}\left(\mathbf{k}_{n}, t^{\prime}\right) \sin \omega_{n}\left(t-t^{\prime}\right)+L\left(\mathbf{k}_{n}, t\right),
$$

where

$$
\begin{aligned}
& L\left(\mathbf{k}_{n}, t\right)=\frac{i \alpha^{2}}{\omega_{n} L^{3}} \sum_{n^{\prime}} \int_{0}^{t} d t^{\prime} \sin \omega_{n}\left(t-t^{\prime}\right) \int_{0}^{t^{\prime}} d t^{\prime \prime} {\left[\phi\left(\mathbf{k}_{n^{\prime}}, t^{\prime \prime}\right) \Omega_{0}\left(\mathbf{k}_{n}, t^{\prime}\right) \Omega_{0}\left(-\mathbf{k}_{n^{\prime}}, t^{\prime \prime}\right)\right.} \\
&\left.-\phi\left(\mathbf{k}_{n^{\prime}}, t^{\prime}-t^{\prime \prime}\right) \Omega_{0}\left(-\mathbf{k}_{n^{\prime}}, t^{\prime}-t^{\prime \prime}\right) \Omega_{0}\left(\mathbf{k}_{n}, t^{\prime}\right)\right] .
\end{aligned}
$$

We now take the statistical expectation value of eq. (B9) with respect to the free states of the heat reservoir, which are assumed to obey a canonical distribution. The matrix elements of $\Omega_{0}\left(\mathbf{k}_{n}, t\right)$ between state $<a \mid$ and $\mid b>$ of the uncoupled reservoir system are written as

$$
<a\left|\Omega_{0}\left(\mathbf{k}_{n}, t\right)\right| b>\equiv\left(\Omega_{r}^{a b}\left(\mathbf{k}_{n}, t\right)+i \Omega_{i}^{a b}\left(\mathbf{k}_{n}, t\right)\right) e^{-i \omega_{a b}^{R} t}
$$

where 


$$
\omega_{a b}^{R}=\omega_{a}^{R}-\omega_{b}^{R}
$$

with $\omega_{a}^{R}$ being the energy of the a-th state of the uncoupled reservoir. Hermeticity of $\Omega$ implies that $\Omega_{r}^{a b}=\Omega_{r}^{b a}$ and $\Omega_{i}^{a b}=-\Omega_{i}^{b a}$. We assume that the diagonal matrix elements are zero

$$
\Omega_{r}^{a a}=0 .
$$

This is equivalent to assuming that there is no time independent force exerted by the heat reservoir on the system. The only nontrivial term for which the statistical expectation value needs to be evaluated is eq. (B16). The expectation value of the first term on the right hand side of eq. (B16) is zero due to eq. (B20). However, we must retain this term in its operator form since we will also want products of it in the final rate equation when evaluating correlation functions.

For $L\left(\mathbf{k}_{n}, t\right)$ in eq. (B16), upon statistical averaging we get

$$
\frac{1}{N} \sum_{a}\left\langle a\left|L\left(\mathbf{k}_{n}, t\right)\right| a\right\rangle e^{-\omega_{a}^{R} / T}=L_{T}^{D}\left(\mathbf{k}_{n}, t\right)+L_{T}^{C}\left(\mathbf{k}_{n}, t\right)
$$

where

$$
\begin{aligned}
& L_{T}^{D}\left(\mathbf{k}_{n}, t\right)=\frac{2 \alpha^{2}}{\omega_{n} N L^{3}} \sum_{a b} e^{-\omega_{a}^{R} / T} \sum_{n^{\prime}} \int_{0}^{t} d t^{\prime} \sin \omega_{n}\left(t-t^{\prime}\right) \int_{0}^{t^{\prime}} d t^{\prime \prime} \sin \omega_{a b}^{R}\left(t^{\prime}-t^{\prime \prime}\right) \phi\left(\mathbf{k}_{n^{\prime}}, t^{\prime \prime}\right) \Omega_{D}^{2}\left(a, b, \mathbf{k}_{n}, \mathbf{k}_{n^{\prime}}\right) \\
& L_{T}^{C}\left(\mathbf{k}_{n}, t\right)=-\frac{2 \alpha^{2}}{\omega_{n} N L^{3}} \sum_{a b} e^{-\omega_{a}^{R} / T} \sum_{n^{\prime}} \int_{0}^{t} d t^{\prime} \sin \omega_{n}\left(t-t^{\prime}\right) \int_{0}^{t^{\prime}} d t^{\prime \prime} \cos \omega_{a b}^{R}\left(t^{\prime}-t^{\prime \prime}\right) \phi\left(\mathbf{k}_{n^{\prime}}, t^{\prime \prime}\right) \Omega_{C}^{2}\left(a, b, \mathbf{k}_{n}, \mathbf{k}_{n^{\prime}}\right)
\end{aligned}
$$

with

$$
\begin{gathered}
N \equiv \sum_{a} e^{-\omega_{a}^{R} / T} \\
\Omega_{D}^{2}\left(a, b, \mathbf{k}_{n}, \mathbf{k}_{n^{\prime}}\right) \equiv \Omega_{r}^{a b}\left(\mathbf{k}_{n}\right) \Omega_{r}^{b a}\left(-\mathbf{k}_{n^{\prime}}\right)-\Omega_{i}^{a b}\left(\mathbf{k}_{n}\right) \Omega_{i}^{b a}\left(-\mathbf{k}_{n^{\prime}}\right),
\end{gathered}
$$

and

$$
\Omega_{C}^{2}\left(a, b, \mathbf{k}_{n}, \mathbf{k}_{n^{\prime}}\right) \equiv \Omega_{r}^{a b}\left(\mathbf{k}_{n}\right) \Omega_{i}^{b a}\left(-\mathbf{k}_{n^{\prime}}\right)+\Omega_{i}^{a b}\left(\mathbf{k}_{n}\right) \Omega_{r}^{b a}\left(-\mathbf{k}_{n^{\prime}}\right) .
$$

The energy levels in the heat reservoir are assumed to be closely spaced so that we can replace the summation over the states by an integration as

$$
\sum_{a b} \rightarrow \int_{0}^{\infty} \rho\left(\omega_{a}^{R}\right) d \omega_{a}^{R} \int_{0}^{\infty} \rho\left(\omega_{b}^{R}\right) d \omega_{b}^{R}
$$

We perform the above energy integration with the variables 


$$
\begin{aligned}
& \omega_{+}^{R} \equiv \frac{\omega_{a}^{R}+\omega_{b}^{R}}{2} \\
& \omega_{-}^{R} \equiv \omega_{a}^{R}-\omega_{b}^{R},
\end{aligned}
$$

so that

$$
\int_{0}^{\infty} d \omega_{a}^{R} \int_{0}^{\infty} d \omega_{b}^{R}=\int_{0}^{\infty} d \omega_{-}^{R} \int_{\frac{\omega_{-}^{R}}{2}}^{\infty} d \omega_{+}^{R}+\int_{-\infty}^{0} d \omega_{-}^{R} \int_{\frac{-\omega_{-}^{R}}{2}}^{\infty} d \omega_{+}^{R} \equiv \int d \tilde{\omega}_{-}^{R} \int d \tilde{\omega}_{+}^{R} .
$$

Eqs. (B22) and (B23) then become respectively

$$
\begin{aligned}
& L_{T}^{D}\left(\mathbf{k}_{n}, t\right)=\frac{-2 \alpha^{2}}{\omega_{n} N L^{3}} \sum_{n^{\prime}} \int d \tilde{\omega}_{-}^{R} \int d \tilde{\omega}_{+}^{R} \rho\left(\tilde{\omega}_{+}^{R}+\frac{\tilde{\omega}_{-}^{R}}{2}\right) \rho\left(\tilde{\omega}_{+}^{R}-\frac{\tilde{\omega}_{-}^{R}}{2}\right) \\
& e^{-\left(\tilde{\omega}_{+}^{R}+\frac{\tilde{\omega}_{-}^{R}}{2}\right) / T} \Omega_{D}^{2}\left(\tilde{\omega}_{+}^{R}+\frac{\tilde{\omega}_{-}^{R}}{2}, \tilde{\omega}_{+}^{R}-\frac{\tilde{\omega}_{-}^{R}}{2}, \mathbf{k}_{n}, \mathbf{k}_{n^{\prime}}\right) \\
& \int_{0}^{t} d \tau\left[\frac{\cos \left(\tilde{\omega}_{-}^{R}+\omega_{n}\right) \frac{\tau}{2} \sin \left(\tilde{\omega}_{-}^{R}-\omega_{n}\right) \frac{\tau}{2}}{\tilde{\omega}_{-}^{R}-\omega_{n}}-\frac{\cos \left(\tilde{\omega}_{-}^{R}-\omega_{n}\right) \frac{\tau}{2} \sin \left(\tilde{\omega}_{-}^{R}+\omega_{n}\right) \frac{\tau}{2}}{\tilde{\omega}_{-}^{R}+\omega_{n}}\right] \phi\left(\mathbf{k}_{n^{\prime}}, t-\tau\right) \\
& L_{T}^{C}\left(\mathbf{k}_{n}, t\right)=\frac{-2 \alpha^{2}}{\omega_{n} N L^{3}} \sum_{n^{\prime}} \int d \tilde{\omega}_{-}^{R} \int d \tilde{\omega}_{+}^{R} \rho\left(\tilde{\omega}_{+}^{R}+\frac{\tilde{\omega}_{-}^{R}}{2}\right) \rho\left(\tilde{\omega}_{+}^{R}-\frac{\tilde{\omega}_{-}^{R}}{2}\right) \\
& e^{-\left(\tilde{\omega}_{+}^{R}+\frac{\tilde{\omega}_{-}^{R}}{2}\right) / T} \Omega_{C}^{2}\left(\tilde{\omega}_{+}^{R}+\frac{\tilde{\omega}_{-}^{R}}{2}, \tilde{\omega}_{+}^{R}-\frac{\tilde{\omega}_{-}^{R}}{2}, \mathbf{k}_{n}, \mathbf{k}_{n^{\prime}}\right) \\
& \int_{0}^{t} d \tau \sin \left(\tilde{\omega}_{-}^{R}+\omega_{n}\right) \frac{\tau}{2} \sin \left(\tilde{\omega}_{-}^{R}-\omega_{n}\right) \frac{\tau}{2}\left[\frac{1}{\tilde{\omega}_{-}^{R}-\omega_{n}}-\frac{1}{\tilde{\omega}_{-}^{R}+\omega_{n}}\right] \phi\left(\mathbf{k}_{n^{\prime}}, t-\tau\right) .
\end{aligned}
$$

Define

$$
B^{I}\left(\omega, \mathbf{k}_{n}, \mathbf{k}_{n^{\prime}}\right) \equiv \int_{0}^{\infty} d \omega^{\prime} \rho\left(\omega^{\prime}+\omega\right) \rho\left(\omega^{\prime}\right) \Omega_{I}^{2}\left(\omega^{\prime}+\omega, \omega^{\prime}, \mathbf{k}_{n}, \mathbf{k}_{n^{\prime}}\right) e^{-\omega^{\prime} / T}
$$

where $I=C$ or $I=D$. We assume $B^{I}$ is a smooth function with respect to $\omega$. Identifying this term in eqs. (B30) and (B31) we observe that due to the oscillatory factor the main contribution to the $\tilde{\omega}_{-}^{R}$ integral is from $\tilde{\omega}_{-}^{R}= \pm \omega_{n}$. Evaluating the $\tilde{\omega}_{-}^{R}$ integration under this approximation, we obtain

$$
\begin{aligned}
& L_{T}^{D}\left(\mathbf{k}_{n}, t\right)=\sum_{n^{\prime}} \Gamma_{n n^{\prime}}^{D} \int_{0}^{t} d t^{\prime} \phi\left(\mathbf{k}_{n^{\prime}}, t^{\prime}\right) \cos \omega_{n}\left(t-t^{\prime}\right) \\
& L_{T}^{C}\left(\mathbf{k}_{n}, t\right)=\sum_{n^{\prime}} \Gamma_{n n^{\prime}}^{C} \int_{0}^{t} d t^{\prime} \phi\left(\mathbf{k}_{n^{\prime}}, t^{\prime}\right) \sin \omega_{n}\left(t-t^{\prime}\right)
\end{aligned}
$$

where

$$
\Gamma_{n n^{\prime}}^{I} \equiv \frac{\pi \alpha^{2}}{\omega_{n} N L^{3}}\left(1-e^{-\omega_{n} / T}\right) B^{I}\left(\omega_{n}, \mathbf{k}_{n}, \mathbf{k}_{n^{\prime}}\right)
$$


with $I=C, D$. Substituting this into eq. (B12) we obtain the equation of motion

$$
\ddot{\phi}\left(\mathbf{k}_{n}, t\right)+\omega_{n}^{2} \phi\left(\mathbf{k}_{n}, t\right)+\sum_{n^{\prime}} \Gamma_{n n^{\prime}}^{D} \dot{\phi}\left(\mathbf{k}_{n^{\prime}}, t\right)+\omega_{n} \sum_{n^{\prime}} \Gamma_{n n^{\prime}}^{C} \phi\left(\mathbf{k}_{n^{\prime}}, t\right)+\frac{\delta V}{\delta \phi\left(-\mathbf{k}_{n}, t\right)}=\eta\left(\mathbf{k}_{n}, t\right),
$$

where $\eta\left(\mathbf{k}_{n}, t\right) \equiv-\alpha \Omega_{0}\left(\mathbf{k}_{n}, t\right)$. Hereafter we will assume that the uncoupled reservoir system is translationally invariant so that

$$
\left\langle\left\langle\Omega_{0}\left(\mathbf{k}_{n}, t\right) \Omega_{0}\left(\mathbf{k}_{n^{\prime}}, t^{\prime}\right)\right\rangle\right\rangle_{T}=\delta_{n,-n^{\prime}}\left\langle\left\langle\Omega_{0}\left(\mathbf{k}_{n}, t\right) \Omega_{0}\left(-\mathbf{k}_{n}, t\right)\right\rangle\right\rangle_{T}
$$

The statistical properties of $\eta\left(\mathbf{k}_{n}, t\right)$ are

$$
\begin{gathered}
\left\langle\left\langle\eta\left(\mathbf{k}_{n}, t\right)\right\rangle\right\rangle_{T}=0 \\
\left\langle\left\langle\eta\left(\mathbf{k}_{n}, t\right) \eta\left(\mathbf{k}_{n^{\prime}}, t^{\prime}\right)\right\rangle\right\rangle_{T}=\delta_{n,-n^{\prime}} \frac{\omega_{n} \Gamma_{n, n}^{D} L^{3}}{\pi}\left(\frac{i P}{t-t^{\prime}}+\pi \delta\left(t-t^{\prime}\right) \operatorname{coth}\left(\frac{\omega_{n}}{2 T}\right)\right) .
\end{gathered}
$$

The first property follows from eq. (B20). The second follows from eqs. (B25), (B26), (B32), and (B35) and using

$$
\int_{0}^{\infty} d \omega e^{i \omega t}=\frac{i P}{t}+\pi \delta(t)
$$

In the high temperature limit, eq. (B39) becomes

$$
\left\langle\left\langle\eta\left(\mathbf{k}_{n}, t\right) \eta\left(-\mathbf{k}_{n}, t^{\prime}\right)\right\rangle\right\rangle_{T \rightarrow \infty} \rightarrow 2 L^{3} \Gamma_{n, n}^{D} T \delta\left(t-t^{\prime}\right),
$$

which agrees with eq. (48). 


\section{REFERENCES}

[1] A. H. Guth, Phys. Rev D23, 347 (1981).

[2] A. H. Guth and S. -Y. Pi, Phys. Rev. Lett. 49, 1110 (1982).

[3] J.M. Bardeen, P.J. Steinhardt, \& M.S. Turner, Phys. Rev. D28, 679 (1983); A. Linde, Phys. Lett. 129B, 177 (1983); R. Brandenberger and R. Kahn, Phys. Rev. D29, 2172 (1984).

[4] G. Smoot et al., Astrophys. J. 396, L1 (1992).

[5] C.L. Bennett, et al., Astrophys. J. 436, 423 (1994).

[6] For a review of inflation please see E.W.Kolb and M.S. Turner, The Early Universe, (Addison-Wesley, New York, 1990); P.J.E.Peebles, Principles of Physical Cosmology, (Princeton, 1993).

[7] G. Mazenko, W. Unruh \& R. Wald, Phys. Rev. D31, 273 (1985).

[8] A. Berera and L. Z. Fang, Phys. Rev. Lett. 74, 1912 (1995).

[9] A. Berera, Phys. Rev. Lett. 75, 3218 (1995).

[10] J. M. Bardeen, Phys. Rev. D22, 1882 (1980).

[11] J. Ford, J. Math Phys. 2, 387 (1961); D. W. Jensen, J. Math Phys. 6, 405 (1965).

[12] H. Callen and T. Welton, Phys. Rev. 83, 34 (1951); M. S. Green, J. Chem. Phys. 19, 1036 (1951); R. Kubo, J. Phys. Soc. Japan 12, 570 (1957).

[13] G. W. Ford, M. Kac, and P. Mazur, J. Math. Phys. 6, 504 (1965); G. W. Ford and M. Kac, J. Stat. Phys. 46, 803 (1987).

[14] I. R. Senitzky, Phys. Rev. 95, 904 (1954); 115, 227 (1959); 119, 670 (1960).

[15] S. Nakajima, Prog. Theor. Phys. 20, 948 (1958).

[16] V. B. Magalinskij, Sov. Phys. JEPT 36, 1381 (1959); A. O. Caldeira and A. J. Leggett, Phys. Rev. Lett. 46, 211 (1981).

[17] R. Benguria and M. Kac, Phys. Rev. Lett. 46, 1 (1981); H. Maassen, J. Stat. Phys. 34, 239 (1984).

[18] B.L. Hu, J. P. Paz and Y. Zhang, Phys. Rev D45, 2843 (1992); B.L. Hu, J. P. Paz and Y. Zhang, Phys. Rev D47, 1576 (1993);

[19] S. Habib and H. E. Kandrup, Phys. Rev. D39, 2871 (1989); Phys. Rev. D46, 5303 (1992).

[20] B. L. Hu, Phys. Lett. 108B, 19 (1982); Phys. Lett. 123B, 189 (1983).

[21] J. M. Cornwall and R. Bruinsma, Phys. Rev. D38, 3146 (1988).

[22] G. Alberi and G. Goggi, Phys. Rep. 74, 2 (1981); K. Goulianos, Phys. Rep. 101, 169 (1983).

[23] M. Gleiser and R. O. Ramos, Phys. Rev. D50, 2441 (1994); A. Hosoya and M. Sakagami, Phys. Rev. D29, 2228 (1984); M. Morikawa, Phys. Rev. D33, 3607 (1986). 